Universidad Nacional Experimental Francisco de Miranda (UNEFM). Santa Ana de Coro. Venezuela

María Gabriela Quezada-Padrón; Darwin Gabriel García-Herrera; Ana Zulema Castro-Salazar; Juan Carlos Erazo-Álvarez

DOI $10.35381 / \mathrm{cm} . v 6 \mathrm{i} 3.391$

\title{
Incidencia del nivel de instrucción de los padres en la iniciación de la lectoescritura
}

\section{Incidence of the level of education of parents in the initiation of literacy}

\author{
María Gabriela Quezada-Padrón \\ mariagabriela.quezada@est.ucacue.edu.ec \\ Universidad Católica de Cuenca, Azogues \\ Ecuador \\ https://orcid.org/0000-0002-1324-2143 \\ Darwin Gabriel García-Herrera \\ dggarciah@ucacue.edu.ec \\ Universidad Católica de Cuenca, Azogues \\ Ecuador \\ https://orcid.org/0000-0001-6813-8100 \\ Ana Zulema Castro-Salazar \\ azcastros@ucacue.edu.ec \\ Universidad Católica de Cuenca, Cuenca \\ Ecuador \\ https://orcid.org/0000-0002-3837-314X \\ Juan Carlos Erazo-Álvarez \\ jcerazo@ucacue.edu.ec \\ Universidad Católica de Cuenca, Cuenca \\ Ecuador \\ https://orcid.org/0000-0001-6480-2270
}

Recibido: 20 de agosto de 2020

Aprobado: 15 de noviembre de 2020 
Universidad Nacional Experimental Francisco de Miranda (UNEFM). Santa Ana de Coro. Venezuela

María Gabriela Quezada-Padrón; Darwin Gabriel García-Herrera; Ana Zulema Castro-Salazar; Juan Carlos Erazo-Álvarez

\title{
RESUMEN
}

El objetivo de la presente investigación fue analizar el impacto del nivel académico de los padres de familia en la iniciación de la lectoescritura de los estudiantes en la Unidad Educativa Miguel Díaz Cueva. Se abordó metodológicamente desde un tipo descriptiva no experimental transversal, el universo muestral estuvo constituido por 50 padres de familia que tienen el rol de representantes de la Unidad Educativa Miguel Díaz Cueva en el nivel inicial I, II y preparatoria, así como 3 docentes. El nivel educativo de los padres de familia de los niños de la Unidad Educativa Miguel Díaz Cueva es uno de los factores que inciden en la iniciación a la lectoescritura en edades tempranas, que cursan niveles educativos de inicial I y II, pero no es el determinante porque existen otros factores inter y externos al entorno escolar que inciden como problemas en el proceso de enseñanza - aprendizaje de los niños/as.

Descriptores: Promoción de la lectura; orientación para la lectura; gestión de la educación. (Palabras tomadas del Tesauro UNESCO).

\begin{abstract}
The objective of this research was to analyze the impact of the academic level of parents on the initiation of literacy by students at the Miguel Díaz Cueva Educational Unit. It was methodologically approached from a descriptive, non-experimental transversal type, the sample universe was constituted by 50 parents who have the role of representatives of the Miguel Díaz Cueva Educational Unit in initial level I, II and preparatory, as well as 3 teachers. The educational level of the parents of the children of the Miguel Díaz Cueva Educational Unit is one of the factors that influence the initiation to literacy at an early age, who attend initial I and II educational levels, but it is not the determining factor because there are other factors inter and external to the school environment that affect as problems in the teaching-learning process of children.
\end{abstract}

Descriptors: Reading promotion; reading guidance; educational management. (Words taken from the UNESCO Thesaurus). 
Universidad Nacional Experimental Francisco de Miranda (UNEFM). Santa Ana de Coro. Venezuela

María Gabriela Quezada-Padrón; Darwin Gabriel García-Herrera; Ana Zulema Castro-Salazar; Juan Carlos Erazo-Álvarez

\section{INTRODUCCIÓN}

El derecho a una educación sostenible e inclusiva es un objetivo trazado por las naciones como un propósito para mejorar la vida de los seres humanos, por eso en las sociedades desarrolladas han perfilado enormes avances en la misión de lograr que los niños asistan a clase, y la cantidad de niños escolarizados se ha incrementado en todo el mundo. Sin embargo, tal como se pone de relieve en el Informe sobre el desarrollo mundial 2018 (Psacharopoulos \& Patrinos, 2018), el aprendizaje no está garantizado, ya que para, alrededor del $50 \%$ de los alumnos escolarizados, no es lo mismo que aprendizaje. Lamentablemente cientos de millones de niños, pese a haber asistido a la escuela, no saben leer ni escribir. En África al sur del Sahara no todos los niños asisten a la escuela, unos 260 millones de niños aún no van a la escuela primaria ni secundaria, casi el $90 \%$ de los alumnos no cuentan con las destrezas mínimas para la lectura y las matemáticas (Banco Mundial, 2018).

El Grupo Banco Mundial (GBM) trabaja con los países para mejorar y organizar sus sistemas educativos, haciendo hincapié en garantizar que todos los niños aprendan. La educación es imprescindible para generar el capital humano que posibilite que las personas y los países prosperen (Banco Mundial, 2018)

En el Ecuador para garantizar la excelencia e inclusividad en la educación se ha protegido este derecho de los niños y niñas por medio de la Constitución de la República del Ecuador que en su carta magna expresa textualmente en su artículo 26 lo siguiente:

“... La educación es un derecho de las personas a lo largo de su vida y un deber ineludible e inexcusable del Estado. Constituye un área prioritaria de la política pública y de la inversión estatal, garantía de la igualdad e inclusión social y condición indispensable para el buen vivir. Las personas, las familias y la sociedad tienen el derecho y la responsabilidad de participar en el proceso educativo." (Asamblea Nacional Constituyente del Ecuador, 2008). 
Universidad Nacional Experimental Francisco de Miranda (UNEFM). Santa Ana de Coro. Venezuela

María Gabriela Quezada-Padrón; Darwin Gabriel García-Herrera; Ana Zulema Castro-Salazar; Juan Carlos Erazo-Álvarez

Así como Garantizar el acceso a la educación a los niños y niñas es imprescindible para dotarlos de herramientas que les faciliten tener libertad de expresión, el Código de la Niñez y la Adolescencia por medio del artículo 59 referente a los Derechos de Participación de los niños (Asamblea Nacional Contituyente del Ecuador, 2013).

Puesto que a medida que los estudiantes van cursando de año básico, el nivel de complejidad se va acrecentando debido a las exigencias que se requieren en la educación superior. Pero es de vital importancia que a edad temprana (Educación Básica) se aprenda a escribir y leer bien, que son actividades necesarias para poder expresarse. Estas asignaturas se vuelven tediosas para el estudiante por la presión de sus representantes, los llamados de atención del docente y las bajas calificaciones en sus tareas, lecciones, en definitiva, un mal rendimiento en la asignatura, por eso la Ley Orgánica de Educación Intercultural nos dice en su artículo 2 literal f) que: “...Los niveles educativos deben adecuarse a ciclos de vida de las personas, a su desarrollo cognitivo, afectivo y psicomotriz, capacidades, ámbito cultural y lingüístico, sus necesidades y las del país, atendiendo de manera particular la igualdad real de grupos poblacionales históricamente excluidos o cuyas desventajas se mantienen vigentes, como son las personas y grupos de atención prioritaria previstos en la Constitución de la República" (Asamblea Nacional Constituyente del Ecuador, 2011).

Cuando existe problemas con el aprendizaje, uno de los factores que influyen en el proceso de aprendizaje es el nivel académico de los padres de familia, que por su condición no colaboran adecuadamente en la educación de sus hijos, en ciertos casos incluso sólo ven a la educación como un mero proceso de la vida y no distinguen la importancia de la misma dentro del desarrollo formativo y cognitivo del estudiante.

Un estudiante con dificultad en lectoescritura no exhibe solamente dificultades en el área de Lengua y Literatura, sino también en el resto de las disciplinas, el retraso lector o escrito no sólo impide el progreso escolar sino que tiene efectos a largo plazo; el fracaso escolar podría también ser el inicio de un fracaso social por los efectos que este puede 
Universidad Nacional Experimental Francisco de Miranda (UNEFM). Santa Ana de Coro. Venezuela

María Gabriela Quezada-Padrón; Darwin Gabriel García-Herrera; Ana Zulema Castro-Salazar; Juan Carlos Erazo-Álvarez

generar en el autoconcepto y autoestima de los estudiantes, teniendo repercusión en el planteamiento de sus metas, aspiraciones, en sus relaciones sociales y en la toma de decisiones concernientes a su futuro académico y profesional. Conociendo el porqué de las dificultades en la adquisición de este proceso, estaremos mejor preparados para comprender los trastornos que se producen en el curso de su adquisición y advertir su aparición, así como favorecer su correcto aprendizaje.

De acuerdo al análisis que se han realizado dentro de los organismos encargados del área pedagógica (Junta académica, Juntas de curso) y los resultados de las pruebas Ser bachiller de la Unidad Educativa Miguel Díaz Cueva, se ha podido determinar en la institución existen muchos niños con un rendimiento escolar bajo asociados a la lectoescritura. Esta problemática ha sido dada a conocer a los padres de familia en asambleas generales, sus respuestas siempre han sido relacionadas a que ellos no tienen mayor conocimiento y que por lo tanto es imposible poder orientarles a sus hijos en el plano académico.

La presente investigación tiene el objetivo de analizar el impacto del nivel académico de los padres de familia en la iniciación de la lectoescritura de los estudiantes en la Unidad Educativa Miguel Díaz Cueva, permitiendo identificar las falencias en otras áreas y poder implementar un plan de acción que busque el desarrollo del estudiante y mejorar la formación de apoyo de los padres de familia.

Además, es importante trabajar en un proyecto de investigación que permita determinar si existe o no relación entre el bajo rendimiento académico de los estudiantes con el nivel de estudio de sus padres, considerando que en los últimos años han existido múltiples variaciones en el currículo ecuatoriano, así como en la sociedad, es decir lo que los padres de familia aprendieron, no es lo mismo que hoy en día se imparte en las aulas escolares, se debe procurar formar y actualizar a los representantes para que de alguna manera puedan orientar adecuadamente a sus representados. 
Universidad Nacional Experimental Francisco de Miranda (UNEFM). Santa Ana de Coro. Venezuela

María Gabriela Quezada-Padrón; Darwin Gabriel García-Herrera; Ana Zulema Castro-Salazar; Juan Carlos Erazo-Álvarez

Esta investigación será un aporte para todos los actores del sistema educativo de la Unidad Educativa Miguel Díaz Cueva, quienes con los datos obtenidos podrán tomar todas las medidas correctivas que permitan mejorar el rendimiento académico de los estudiantes mediante el trabajo conjunto con los padres de familia.

Es trascendental que se analice cada uno de los factores que inciden en el proceso de enseñanza-aprendizaje de los niños de la Unidad Educativa Miguel Díaz Cueva, para que se pueda intervenir de manera eficaz desde edades tempranas y fortalecer a cada uno de las personas inmersas en la educación de los niños.

\section{Referencial teórico}

\section{La lectoescritura}

La lectoescritura se admite como la manera más compleja que posee el hombre para comunicarse y a su vez es el medio por excelencia de registro de las variaciones culturales y técnicas de la humanidad. Según la autora (Calvo, 2011), manifiesta que la familia ha derivado en la escuela, la responsabilidad exlusiva de la enseñanza la lectoescritura. Según (Ferreiro \& Teberosky, 1979), "el niño adquiere la lengua escrita en cuatro etapas: presilábica, silábica, silábica-alfabética y alfabética.

\section{La Familia y su influencia en la Educación de sus hijos}

La familia es el eje principal para el desarrollo óptimo del individuo y por ende de la sociedad. Los patrones marcados por la familia influiran directamente en la manera en cómo el individuo ejerza su vida, la orientación religiosa que tenga, los valores que practique, las costumbres que presente, etc. La familia es una institución inmersa en la sociedad donde se nutre de sus valores, ideas, actitudes, sentimientos y les otorga características que son frecuentes en todas las familias que la integran (Caira, 2014). Existen peculiaridades que hacen que cada familia sea diferente, al igual que cada uno de sus miembros. Diferiran en su particular estilo de educar, criar a sus hijos, infundir 
Universidad Nacional Experimental Francisco de Miranda (UNEFM). Santa Ana de Coro. Venezuela

María Gabriela Quezada-Padrón; Darwin Gabriel García-Herrera; Ana Zulema Castro-Salazar; Juan Carlos Erazo-Álvarez

valores, mismos que son el resulatado del contexto socio-histórico en el que se desenvuelven, de las prácticas observadas de las figuras parentales y de la dinámica del grupo familiar. Los padres son los primeros agentes que interceden en el proceso de socialización de sus hijos, su objetivo principal es el de ayudarlos a insertarse armoniosamente en el mundo físico y social (Caira, 2014).

Al respecto, la investigación de (Calvo, 2011) señala que "a través de la familia el hijo es introducido en la cultura de su tiempo y de su medio. La relación de este con otros individuos será más adecuada si el apoyo de sus padres ha sido favorable, y ello a la larga les permitirá integrarse y adaptarse en un grupo social más amplio"

Son diversos los factores que influyen en el estilo de crianza empleado por los padres en la educaciòn de sus hijos. En este sentido, dentro del grupo familiar los padres cumplen roles y funciones importantes en la formación psicológica del ser humano predestinadas a mejorar su adaptaciòn, funcionamiento y desarrollo dentro de la sociedad.

Una de las funciones de la familia se situa que en los hogares se desarrollen lazos de afecto y solidaridad, dentro de la cual se trasfieren los valores que nutren y enriquecen la vida de los individuos; se congregan para asignar los recursos que seran dirigidos a satisfacer los requerimientos de sus miembros, realizando la división del trabajo con arreglo a las normas culturales y de acuerdo con la edad, el sexo y el vínculo de sus integrantes, y se toman las decisiones relativas a los eventos vitales de relevancia en el ámbito demográfico que estructuran y marcan la trayectoria de vida de las personas (Caira, 2014).

Mediante las interacciones sociales dentro del grupo familiar se propone una serie de interacciones sociales que van a influir de manera decisiva en el curso del desarrollo.

Por otra parte, (Rodrigo, M. y Palacios, J., 1998), proponen 4 funciones esenciales:

a. Afirmar la conservación y el sano crecimiento físico de todos los integrantes de la familia. 
Universidad Nacional Experimental Francisco de Miranda (UNEFM). Santa Ana de Coro. Venezuela

María Gabriela Quezada-Padrón; Darwin Gabriel García-Herrera; Ana Zulema Castro-Salazar; Juan Carlos Erazo-Álvarez

b. Ofrecer un clima de afecto y sostén, sin los cuales un adecuado desarrollo psicológico sano no sería posible.

c. Suministrar a los hijos la motivación que haga de ellos seres con capacidad para relacionarse competentemente con su entorno físico y social, así como para responder a las demandas y exigencias planteadas por su adaptación al mundo en el que les tocó vivir.

d. Tomar decisiones con respecto a la apertura hacia otros contextos educativos y sociales que van a compartir con la familia la tarea de educar a los hijos (Rodrigo, M. y Palacios, J., 1998).

Dentro de los roles de la familia està el atribuir estilos educativos de crianza, que han recibido diversos calificativos tales como: estilos educativos paternos o familiares, estilos educativos parentales, estilos parentales o de relación parental, dependiendo por lo general de si la perspectiva utilizada es pedagógica, sociológica, antropológica o psicológica. A oposición a la simple descripción de experiencias particulares utilizadas por los padres con sus hijos, La concepción de estilos alude a la complejidad de la crianza en la cual están implicadas no solo las acciones sino también las emociones, las actitudes y los valores.

El tomar conciencia de esta complejidad suministra la predicción del tipo de influjo que ejerce la crianza sobre el futuro comportamiento de los hijos (Calvo, 2011). Al hablar de estilos educativos de crianza debemos referirnos a las tendencias globales de comportamiento y las practicas más habituales, con ello no se intenta decir que los padres manejen siempre los mismos estilos con todos sus hijos ni en todas los escenarios, sino que los padres, dentro de un continuo más o menos amplios estilos de crianza, opten con mayor generalidad determinados modelos educativas (Ceballos, E. y Rodrigo, M., 1998). Una de las variables de presente estudio es el estilo educativo de crianza, es definido como el agregado de esquemas de acción, similares entre sí, que se refrendan en el tiempo, en familias diferentes y constituyen un rasgo cultural. Por consiguiente, el patrón 
Universidad Nacional Experimental Francisco de Miranda (UNEFM). Santa Ana de Coro. Venezuela

María Gabriela Quezada-Padrón; Darwin Gabriel García-Herrera; Ana Zulema Castro-Salazar; Juan Carlos Erazo-Álvarez

de crianza es el estilo que adopta el sistema familiar, a través de los padres, en la educación de sus hijos (Caira, 2014).

Para (Calvo, 2011) los estilos de crianza comprenden el conjunto de patrones de actuación que asumen los padres en el proceso de formación y crecimiento de sus hijos, abarcando diversos estilos basados en la experiencia de los propios padres y en el nivel de información que estos manejan sobre los comportamientos que deben adoptar.

Para fines de esta investigación, se define al estilo educativo de crianza como, el agregado de acciones, sentimientos, actitudes y comportamientos que los padres presentan hacia los hijos y que, tomados como un todo, componen un clima emocional favorable o desfavorable para el desarrollo de los mismos (Darling \& Steinberg, 2015). Muchas veces las personas se cuestionan si hay algún estilo particular de crianza que surta a los hijos el ambiente necesario para engrandecer sus vidas. También, se cuestionan en qué momento se debe corregir, felicitar y estimular a que se logre algo o, cuando dimitir de su esfuerzo.

Siempre se cuestiona por no saber cuándo se está disciplinando demasiado o muy poco. Todos estas interrogantes son importantes para la relación diaria en la familia. Previo a considerar cual estilo educativo de crianza es el más apropiado para la familia, se debe indagar cuál es el propósito y el rol de la familia. Un punto en común entre todos los teóricos y campos científicos como la psicología, la educación, la psiquiatría, la sociología, entre otros es que la familia tiene como función el cumplimiento de ciertas tareas, de las cuales hablaremos en líneas generales:

1. La capacidad de nutrir tanto física como emocionalmente a todos los miembros.

2. Fundar y conservar fronteras apropiadas entre cada uno de los miembros de la familia y entre cada generación. Es decir que cada integrante debe sentirse como único y con un rol determinado, sino que cada miembro de la familia debe poder reconocer las diferencias y las reglas entre cada generación familiar.

3. Ofrecer en la nueva generación valores familiares y culturales. 
Universidad Nacional Experimental Francisco de Miranda (UNEFM). Santa Ana de Coro. Venezuela

María Gabriela Quezada-Padrón; Darwin Gabriel García-Herrera; Ana Zulema Castro-Salazar; Juan Carlos Erazo-Álvarez

4. Promover la independencia de los hijos, aminorando cualquier tipo de apego o dependencia.

5. Proveer las herramientas necesarias para la resolución de problemas.

6.

\section{El rendimiento escolar}

Además, en esta investigación es necesario conocer sobre el concepto del rendimiento académico, que se define como:

El producto de la asimilación del contenido de los programas de estudio, expresado en calificaciones dentro de una escala convencional. En otras palabras, se refiere al resultado cuantitativo que se obtiene en el proceso de aprendizaje de conocimientos, conforme a las evaluaciones que realiza el docente mediante pruebas objetivas y otras actividades complementarias (Salinas, 2017).

Siendo el Rendimiento Académico cuantificable, determina el nivel de conocimiento logrado, y es tomado como único criterio para medir el éxito o fracaso escolar a través de un sistema de calificaciones que en caso de nuestro país va de 0 a 20 y el sistema de letras que van desde a $C$ hasta $A D$ para expresar el nivel alcanzado Inicio, Proceso, Logro esperado y Logro destacado.

En realidad, el Rendimiento Académico expresa el resultado de las diferentes y complejas fases del proceso educativo, una de las metas en las que confluyen todos los esfuerzos y todas las iniciativas de las autoridades educativas, docentes, representantes y alumnos. No nos referimos a cuánto material han memorizado los estudiantes sino de cuánto han interiorizado realmente, exteriorizandolo en su manera de sentir, de resolver los problemas y hacer o utilizar cosas cursadas.

En este sentido, el Rendimiento Académico se discurre como el conjunto de evoluciones operadas en el educando, a través del proceso enseñanza-aprendizaje, que se manifiesta mediante el desarrollo y incremento de la personalidad en formación. El Rendimiento Académico, sintetiza la acción del proceso educativo, no sólo en el aspecto cognoscitivo logrado por el educando, sino también en el conjunto de habilidades, destrezas, 
Universidad Nacional Experimental Francisco de Miranda (UNEFM). Santa Ana de Coro. Venezuela

María Gabriela Quezada-Padrón; Darwin Gabriel García-Herrera; Ana Zulema Castro-Salazar; Juan Carlos Erazo-Álvarez

aptitudes, ideales, intereses, Etc. Con esta síntesis están los esfuerzos de la sociedad, del profesor y del proceso enseñanza-aprendizaje. El profesor es el responsable en gran parte del rendimiento escolar. Intervienen en éste una serie de factores, entre ellos, la metodología del profesor, el aspecto individual del alumno, el apoyo familiar, la situación social, entre otros.

En consecuencia, el Rendimiento Académico no se puede sujetar a la suma de apreciaciones como fruto de la exploración de conocimientos al que ha sido subordinado el alumno, para comprobar e inspeccionar su aprendizaje. Se refiere a una serie de cambios conductuales susodichos de la acción educativa, que trasciende y se sitúa en el campo de la comprensión y sobre todo en los que se encuentran implicados los hábitos, destrezas, habilidades y otros modos de ser y hacer frente a diferentes escenarios de la vida cotidiana. En tal sentido, el rendimiento académico es una medida de las capacidades del escolar, que expresa lo que éste ha asimilado a lo largo del proceso pedagógico.

Los tipos de rendimientos académicos, según (Edel, 2013) son:

- Rendimiento Individual: Se manifiesta en la ganancia de conocimientos, experiencias, hábitos, destrezas, habilidades, actitudes, aspiraciones, etc.; lo que permitirá al educador tomar decisiones pedagógicas posteriores. Los aspectos de rendimiento individual, se apoyan en la explotación de los conocimientos y de los hábitos culturales, campo cognoscitivo o intelectual.

- Rendimiento Específico: Es el producto que se da en la resolución de los problemas personales, progreso en la vida profesional, familiar y social que se les presentan. En este producto la ejecución de la valoración es más dificultosa, por cuanto si se valora la vida afectiva del educando, se debe considerar su conducta parceladamente: sus relaciones con el maestro, con las cosas, consigo mismo, con su modo de vida y con sus semejantes. 
Universidad Nacional Experimental Francisco de Miranda (UNEFM). Santa Ana de Coro. Venezuela

María Gabriela Quezada-Padrón; Darwin Gabriel García-Herrera; Ana Zulema Castro-Salazar; Juan Carlos Erazo-Álvarez

- Rendimiento Social: Expresa la sociabilidad, la entrega mutua, el grado de contribución, la coincidencia de caracteres, la participación activa y solidaria de los estudiantes en la labor escolar: y sirve para que el profesor mida y calcule los contenidos programáticos, la metodología de la enseñanza y el sistema de evaluación. El rendimiento social es una guía importante del sistema enseñanzaaprendizaje, ya que la expresión medio de comprensión del grupo es primordial para que el profesor gradúe y dosifique los contenidos programados en su metodología de enseñanza.

Existen diferentes tipos de rendimiento escolar, que identifican otros autores, los cuales se dan según el lugar donde se desenvuelven los estudiantes. Siendo estos:

\section{Factores positivos del rendimiento académico}

\section{Factores internos o endógenos}

a. Factores biológicos: Uno de los factores que intervienen en el rendimiento académico es el biologico en el que interviene todo nuestro cuerpo, particularmente el sistema nervioso en general y el cerebro, debe estar en perfectas condiciones de salud. Es decir, el estado de salud, el estado de nutrición, el estado anatómico y fisiológico de todos los órganos, aparatos y sistemas del individuo.

b. Factores psicológicos: En este factor, actúa lo psíquico, primariamente la atención, la voluntad, la memoria, la inteligencia, la conciencia, el pensamiento, la afectividad; por lo que es significativo gozar de buena salud.

\section{Factores externos o exógenos}

Son los factores que vienen del exterior:

a. Factores sociales: Los factores sociales son el agregado de unidades sociales en que se desarrolla el alumno. Por lo tanto, para que exista un correcto rendimiento es sustancial que el alumno disfrute de buenas relaciones sociales: 
Universidad Nacional Experimental Francisco de Miranda (UNEFM). Santa Ana de Coro. Venezuela

María Gabriela Quezada-Padrón; Darwin Gabriel García-Herrera; Ana Zulema Castro-Salazar; Juan Carlos Erazo-Álvarez

Hogar al cual pertenece, clase social en la cual se desenvuelve, modo de vida que le es usual, tipo de trabajo que realiza, práctica social concreta que efectúa, nivel educacional que posee, grado y calidad de estimulación socio-cultural a la que es expuesto, etc.

b. Factores pedagógicos: Son la influencia, el educador, el currículo, la metodología de la enseñanza, el sistema de evaluación, los recursos didácticos, el local escolar, el mobiliario escolar, el horario de trabajo, la manera de estudiar, etc. En ocasiones el rendimiento se ve determinado por el número excesivo de alumnos, aulas mal acondicionadas, pedagogos poco competentes o demasiado autoritarios, programas curriculares no adaptados a la realidad del alumnado. Todo ello favorece a que el alumno no rinda de acuerdo con su capacidad.

c. Factores ambientales: Dentro de las condiciones ambientales, se considera también la iluminación, la ventilación, la temperatura, los ruidos, el ambiente familiar. La estimulación cognoscitiva, sobre un alumno sano, bien alimentado y sin problemas, determinará como respuesta: buen rendimiento escolar; y sobre un alumno enfermo, mal alimentado y con problemas, determinará como respuesta: un mal rendimiento escolar. El tipo de clima, la existencia de parásitos y gérmenes patógenos, la existencia de sustancias tóxicas que contaminan el agua, el suelo y la atmósfera, etc. Por lo tanto, podemos decir que existen muchos factores que influyen en el rendimiento escolar, unos que pertenecen o se encuentran en el mismo individuo, llamados endógenos y otros que pertenecen o se encuentran en el mundo circundante; los que se refieren al mundo exterior son llamados exógenos. 
Universidad Nacional Experimental Francisco de Miranda (UNEFM). Santa Ana de Coro. Venezuela

María Gabriela Quezada-Padrón; Darwin Gabriel García-Herrera; Ana Zulema Castro-Salazar; Juan Carlos Erazo-Álvarez

\section{Factores negativos del rendimiento académico}

Entre los factores negativos que inciden en el rendimiento escolar tenemos:

\section{Factores endógenos}

- Problemas genéticos.

- Anomalías cromosómicas.

- Infecciones, accidentes, intoxicaciones, disminución de oxígeno, enfermedades, etc.

\section{Factores exógenos}

- Problemas del hogar: Desorden familiar, deficiente nivel educativo de la familia, etc.

- Problemas de la sociedad: La injusticia social, el alcoholismo, tabaquismo, etc.

- Problemas de escuela: Improvisacion docente, currículo deficiente, etc.

Así como el rendimiento escolar tiene factores positivos, también tiene factores negativos que inciden negativamente en los alumnos, así tenemos:

La baja autoestima.

- La falta de un adecuado ambiente familiar.

- Contrariedades de interacción social.

- Adiccion a alguna sustancia toxica que daña el organismo de los alumnos.

Mediante investigaciones realizadas para determinar cuáles son las causas que influyen en el bajo rendimiento del alumno se presenta continuación las que se consideran importantes:

a. Los padres: Deberian asumir el verdadero rol de educación, evitando el distanciamiento con los profesores. La misiòn del colegio debe ser inquebrantable en orientar a los padres e hijos, a travez de charlas respecto a la importancia de una adecuada relacion entre el medio familiar y escolar. Es muy preocupante que padres y profesores se muestran como auténticos extraños, los unos para los otros. 
Universidad Nacional Experimental Francisco de Miranda (UNEFM). Santa Ana de Coro. Venezuela

María Gabriela Quezada-Padrón; Darwin Gabriel García-Herrera; Ana Zulema Castro-Salazar; Juan Carlos Erazo-Álvarez

b. Docentes: El docente es la base y la figura a la cual el estudiante quiere seguir, para el efecto se debe tener en cuenta el uso de estrategias innovadoras que consientan el mejoramiento del rendimiento escolar.

c. Autoestima. Es primordial el desarrollo de una adecuada autoestima, con una predisposiciòn que le permita afrontar con éxito las dificultades escolares. Se entiende por autoestima, la impresión de verse bueno y valioso que se concrete a su vez en confianza y seguridad de sí mismo.

d. Relación de los padres con los docentes. Por diversas causas no siempre las relaciones padres-docentes se mantienen en el justo término que pudiese anhelar, en ocasiones son los padres los que no están de acuerdo con los métodos educativos de los docentes, ya sea por su forma de actuación o bien son los docentes quienes desconfían de los padres. Es muy común que los disconformes sean los familiares del estudiante, para quienes no siempre el maestro es lo suficiente competente, se suele acusar de negligente, de incompetente, etc. Estas desconfianzas mutuas podrían enmendarse con el diálogo con las debidas condiciones y no de forma imperativa.

\section{METODOLOGÍA}

Se abordó metodológicamente desde un tipo descriptiva no experimental transversal, el universo muestral estuvo constituido por 50 padres de familia que tienen el rol de representantes de la Unidad Educativa Miguel Díaz Cueva en el nivel inicial I, II y preparatoria, así como 3 docentes. Se utilizó como tecnicas de recolección de datos la encuesta y un cuestionario en escala Likert, siendo validado por juicio de expertos y calculo de Alfa de Cronbach en 0,87 siendo confiable para su aplicación, este fue empleado para compilar la información de los padres. Mientras que la entrevista sirvió para recopilar lo aportado por los docentes. Los datos obtenidos se analizaron 
Universidad Nacional Experimental Francisco de Miranda (UNEFM). Santa Ana de Coro. Venezuela

María Gabriela Quezada-Padrón; Darwin Gabriel García-Herrera; Ana Zulema Castro-Salazar; Juan Carlos Erazo-Álvarez

estadisticamente desde estadistica descriptiva, mientras que la entrevista fue analizada en razon del análisis de contenido.

\section{RESULTADOS}

\section{Docentes}

Los docentes mencionan que realizan actividades de motivación con sus estudiantes antes de iniciar la clase como una manera de introducir la temática a enseñarse según la planificación curricular y ajustando las necesidades educativas de los niños/as.

Que, la influencia del nivel educativo de los padres en el aprendizaje de los niños tiene mucha importancia, pero consideran que la dedicación que le pongan en el aprendizaje y más aún la dedicación al niño/a tiene un significado superior e influyente en el proceso de aprendizaje.

Es por eso que, enviándoles actividades divertidas, acorde a su edad como Pictogramas para que asocien las imágenes con las palabras, rotular objetos con sus respectivos nombres y utilizando estrategias y métodos innovadores, permite que estos docentes incentiven a sus alumnos para que lean o escriban en clases y en sus hogares, de una manera más eficiente.

La metodología que los docentes de la Unidad Educativa Miguel Díaz Cueva utilizan son propuestas de actividades que potencien el desarrollo del lenguaje y les permitan exteriorizar sus pensamientos, ideas, deseos, emociones, vivencia; Metodología Alfabética, Conciencia Fonológica, Semántica, Sintáctica; y lectura de pictogramas y por medio de la lectura de cuentos, rimas, retahílas y trabalenguas. Son todos estas las metodologías utilizadas en el proceso de enseñanza - aprendizaje en la iniciación a la lectoescritura de los niños de educación inicial.

Las áreas de aprendizaje como razonamiento lógico matemático, comprensión oral y escrita y direccionalidad, son las que presentan mayores dificultades en el proceso enseñanza - aprendizaje del grupo de niños, niñas que tienen bajo su responsabilidad 
Universidad Nacional Experimental Francisco de Miranda (UNEFM). Santa Ana de Coro. Venezuela

María Gabriela Quezada-Padrón; Darwin Gabriel García-Herrera; Ana Zulema Castro-Salazar; Juan Carlos Erazo-Álvarez

Los docentes de la Unidad Educativa Miguel Díaz Cueva mencionan que están capacitados para identificar si sus alumnos padecen de algún trastorno de comunicación y problemas de aprendizaje. Tales como: Dificultades en la pronunciación, Trastorno del lenguaje, Trastorno de la articulación del habla dislalia, Trastorno del ritmo y la fluencia tartamudez o espasmofemia

Los docentes reconocen que el $25 \%$ de los niños de su aula presentan características de algún trastorno de comunicación y problemas de aprendizaje, calculando en números un promedio de entre 8, 5 y 4 estudiantes en cada aula.

Los padres de familia en este nivel educativo de sus hijos/as si ayudan con las tareas y fortalecen lo aprendido en clases en sus hogares, es decir, que esto sucedo por el nivel de conocimientos básicos que se requieren en esta etapa escolar y por las edades tempranas que tienen los niños/as.

El desarrollo del lenguaje, su madurez y el apoyo que los padres les brindan, el entorno familiar, la cantidad de estímulos recibidos durante el desarrollo tanto en el embarazo y el crecimiento son los factores que intervienen en el aprendizaje de la lectoescritura de los alumnos de la Unidad Educativa Miguel Díaz Cueva.

El apoyo de los padres, siempre rinde de mejor manera, esto se ve reflejado en su seguridad emocional, madurez, físico, social, emocional, tiene mucha importancia en el entorno familiar en el aprendizaje y en la manera de comunicarse de los niños/as.

Los niños maltratados tanto físicos y psicológicos por parte de los padres son una de las causas para que los mismos presenten alguna dificultad de aprendizaje o comunicación, porque la mayoría de los padres no saben cómo enseñar y pierden la paciencia, siendo esta problemática una evidencia notaria y su influencia en el aprendizaje y comportamiento de los niños/as cuando son afectados.

Los docentes de los niveles de educación inicial de la Unidad Educativa Miguel Díaz Cueva, pueden identificar los signos y síntomas de algún trastorno de aprendizaje con relación a la lectoescritura como dislexia, disgrafia y discalculia, pero no están 
Universidad Nacional Experimental Francisco de Miranda (UNEFM). Santa Ana de Coro. Venezuela

María Gabriela Quezada-Padrón; Darwin Gabriel García-Herrera; Ana Zulema Castro-Salazar; Juan Carlos Erazo-Álvarez

capacitados para diagnosticar, debido a que de los tres docentes entrevistados uno ellos es profesional en parvularia y desconoce temas relacionados con esta temática.

A continuación, en la tabla 1 se puede identificar que la sumatoria de la instrucción académica de los padres encuestados entre básica o menos y bachillerato tienen un porcentaje alto y significativo del $76 \%$, demostrando que este nivel de instrucción académica tiene relevancia en la etapa de educación inicial de los niños/as, mientras que en menor porcentaje de $20 \%$ encontramos padres con títulos de tercer nivel y un $10 \%$ con título de cuarto nivel.

\section{Tabla 1.}

Instrucción académica.

\begin{tabular}{cccccc}
\hline & Instrucción & Frecuencia & Porcentaje & $\begin{array}{c}\text { Porcentaje } \\
\text { válido }\end{array}$ & $\begin{array}{c}\text { Porcentaje } \\
\text { acumulado }\end{array}$ \\
\hline \multirow{4}{*}{ Válido } & 1 & 2,0 & 2,0 & 2,0 \\
& Bachillerato & 18 & 36,0 & 36,0 & 38,0 \\
& Básica o & 20 & 40,0 & 40,0 & 78,0 \\
& menos & 1 & 2,0 & 2,0 & 80,0 \\
& Cuarto Nivel & 10 & 20,0 & 20,0 & 100,0 \\
\hline Tercer Nivel & Total & 50 & 100,0 & 100,0 & \\
\hline
\end{tabular}

Fuente: Elaboración propia.

Otro factor que incide en el proceso de iniciación a la lectoescritura en los niños de la Unidad Educativa Miguel Díaz Cueva, lo podemos observar en la tabla 2, que demuestra la influencia de la persona que ayudan con las tareas de la escuela a los niños/as donde la mamá es la personas que más los ayudan con el $80 \%$, mientras que el papá lo hace en un porcentaje del $6 \%$ y otros miembros de la familia que ayudan a los niños son las abuelitas, tías y hermanos con el $2 \%$ cada uno. 
CIENCIAMATRIA

Revista Interdisciplinaria de Humanidades, Educación, Ciencia y Tecnología

Año VI. Vol. VI. N³. Edición Especial III. 2020

Hecho el depósito de ley: pp201602FA4721

ISSN-L: 2542-3029; ISSN: 2610-802X

Universidad Nacional Experimental Francisco de Miranda (UNEFM). Santa Ana de Coro. Venezuela

María Gabriela Quezada-Padrón; Darwin Gabriel García-Herrera; Ana Zulema Castro-Salazar; Juan Carlos Erazo-Álvarez

Tabla 2.

¿Quiénes ayudan con las tareas de la escuela a sus hijos?.

\begin{tabular}{cccccc}
\hline & Frecuencia & Porcentaje & $\begin{array}{c}\text { Porcentaje } \\
\text { válido }\end{array}$ & $\begin{array}{c}\text { Porcentaje } \\
\text { acumulado }\end{array}$ \\
\hline \multirow{4}{*}{ Válido } & Abuela & 1 & 2,0 & 2,0 & 2,0 \\
& 3 & 6,0 & 6,0 & 8,0 \\
& Abuela y tía & 1 & 2,0 & 2,0 & 10,0 \\
& Hermanos & 1 & 2,0 & 2,0 & 12,0 \\
& Mamá & 40 & 80,0 & 80,0 & 92,0 \\
& Ninguno & 1 & 2,0 & 2,0 & 94,0 \\
& Papá & 3 & 6,0 & 6,0 & 100,0 \\
\hline
\end{tabular}

Fuente: Elaboración propia.

Es importante identificar el tipo de apoyo que recibe el niño/a con relación a su educación, sea esta de refuerzo o llenar los vacíos que no entienden en la institución educativa, es así que en la tabla 3, la mayoría de los padres encuestados con un 92\% mencionaron que son ellos mismos quienes les explica las tareas a sus hijos/as, el $4 \%$ buscan apoyo en el docente y respectivamente con el $2 \%$ dejan que sus hijos estudien solos y no pagan clases particulares

Tabla 3.

¿Cómo ofrece su apoyo a la educación de su hijo/a?

\begin{tabular}{cccccc}
\hline & Frecuencia & Porcentaje & $\begin{array}{c}\text { Porcentaje } \\
\text { válido }\end{array}$ & $\begin{array}{c}\text { Porcentaje } \\
\text { acumulado }\end{array}$ \\
\hline Válido & 1 & 2,0 & 2,0 & 2,0 \\
& $\begin{array}{c}\text { Busca apoyo } \\
\text { de la docente } \\
\text { Deja que su } \\
\text { hijo estudie } \\
\text { por su cuenta } \\
\begin{array}{c}\text { Usted le } \\
\text { explica las } \\
\text { tareas }\end{array}\end{array}$ & 2 & 4,0 & 4,0 & 6,0 \\
Total & 46 & 2,0 & 2,0 & 8,0 \\
\hline
\end{tabular}

Fuente: Elaboración propia. 
Universidad Nacional Experimental Francisco de Miranda (UNEFM). Santa Ana de Coro. Venezuela

María Gabriela Quezada-Padrón; Darwin Gabriel García-Herrera; Ana Zulema Castro-Salazar; Juan Carlos Erazo-Álvarez

La tabla 4 muestra la regularidad con la que los padres familiarizan, incentiva, instruyen a la iniciación a la lectura de libros o textos a sus hijos es el punto determinante para influenciarlos y apoyar en la educación de los mismos, por eso se puede observar en la tabla 4 que los encuestados en un $54 \%$ pocas veces les leen libros o textos a sus hijos, otros con el $34 \%$ dicen leerles con frecuencia, y con el $10 \%$ dicen que lo realizan muy frecuentemente.

Tabla 4.

¿Le lee con regularidad libros o textos a sus hijos como iniciación a la lectoescritura?

\begin{tabular}{cccccc}
\hline & Frecuencia & Porcentaje & $\begin{array}{c}\text { Porcentaje } \\
\text { válido }\end{array}$ & $\begin{array}{c}\text { Porcentaje } \\
\text { acumulado }\end{array}$ \\
\hline \multirow{4}{*}{ Válido } & & 1 & 2,0 & 2,0 & 2,0 \\
& Frecuentemente & 17 & 34,0 & 34,0 & 36,0 \\
& Muy Frecuentemente & 5 & 10,0 & 10,0 & 46,0 \\
\cline { 2 - 5 } & Pocas veces & 27 & 54,0 & 54,0 & 100,0 \\
\hline
\end{tabular}

Fuente: Elaboración propia. 
María Gabriela Quezada-Padrón; Darwin Gabriel García-Herrera; Ana Zulema Castro-Salazar; Juan Carlos Erazo-Álvarez

\section{PROPUESTA}

Considerando que el apoyo e involucramiento de los padres en el proceso de aprendizaje de sus hijos es fundamental, y esto està sustentado en este estudio planteamos una propuesta para un acompañamiento màs cercano y consiente por parte de sus padres 0 cuidadores.

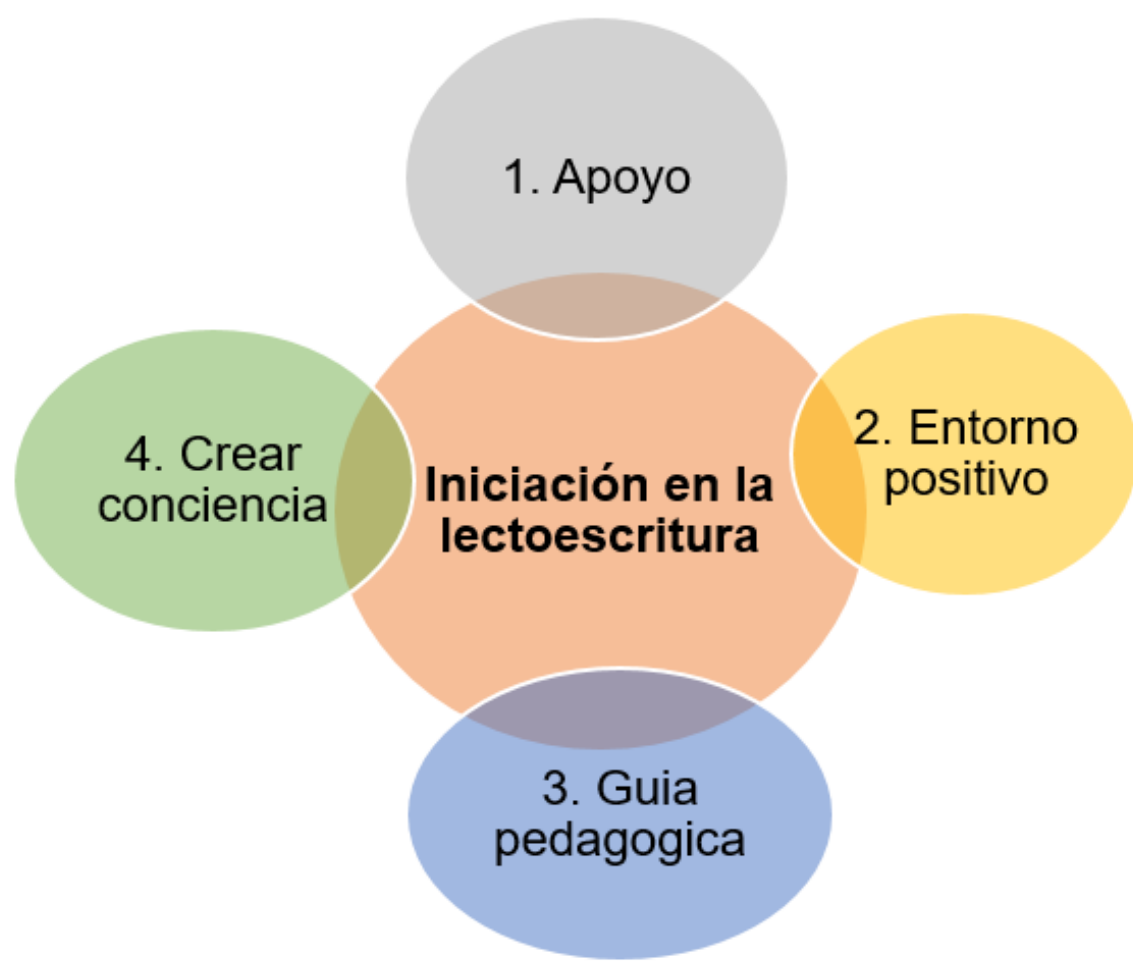

Figura 1. Propuesta de mejoras para la iniciación de la lectoescritura.

Fuente: Elaboración propia. 
Universidad Nacional Experimental Francisco de Miranda (UNEFM). Santa Ana de Coro. Venezuela

María Gabriela Quezada-Padrón; Darwin Gabriel García-Herrera; Ana Zulema Castro-Salazar; Juan Carlos Erazo-Álvarez

1. Apoyo. - Se requiere del apoyo frecuente a los padres de familia mediante notas explicativas, amigables y con términos sencillos por parte de los docentes, para que puedan ayudar con la educación de sus hijos

2. Crear. - Uno de los factores más determinantes es el ambiente en el hogar, por lo tanto, es imprescindible crear entornos positivos, no violentos para los niños/as tanto en el entorno escolar y el familiar

3. Guía pedagógica. - Establecer una guía psicopedagógica que apoye en la práctica de la lectoescritura tanto en el hogar como en el entorno escolar

4. Crear conciencia. - Se propone sensibilizar a los padres de familia mediante charlas, visitas y talleres que les permita convertirse en actores principales de la educación de sus hijos/as, resaltando la importancia de ejercer adecuadamente su rol.

\section{CONCLUSIONES}

A partir de la evidencia disponible en la encuesta, entrevista y observación en la institución educativa objeto de estudio, se demostró la responsabilidad que tiene la familia en la calidad de la educación de sus hijos, es así que los objetivos trazados en esta investigación se pudieron cumplir.

El nivel educativo de los padres de familia de los niños de la Unidad Educativa Miguel Díaz Cueva es uno de los factores que inciden en la iniciación a la lectoescritura en edades tempranas, que cursan niveles educativos de inicial I y II, pero no es el determinante porque existen otros factores inter y externos al entorno escolar que inciden como problemas en el proceso de enseñanza - aprendizaje de los niños/as.

Además, otro de los factores que tiene relevancia es la disposición y dedicación en la educación de los niños/as, esto hace que tengan problemas de lectoescritura, así como también el tiempo que los padres le dediquen y enseñen a leer textos o libros a sus hijos, siendo la lectura una costumbre que se puede adquirir por la práctica. 
Universidad Nacional Experimental Francisco de Miranda (UNEFM). Santa Ana de Coro. Venezuela

María Gabriela Quezada-Padrón; Darwin Gabriel García-Herrera; Ana Zulema Castro-Salazar; Juan Carlos Erazo-Álvarez

En conclusión, es evidente la importancia que tiene la participación de los padres en las escuelas de sus hijos/as, que estos se sientan acogidos y que se les toma en cuenta; por lo tanto, es muy necesario la formación de los maestros/as y futuros maestros/as en cómo afrontar la participación de los padres en la escuela, cómo facilitarla, cuál debe ser su actitud.

Esta preparación hará posible que se lleve a la práctica esta colaboración y buena relación de los padres, madres y maestros/as y que los docentes haciendo posibles resultados positivos para todos, no solo para los niños/as, sino también para la familia y ello/as mismos y mismas. Porque la educación es cosa de todos y todos debemos hacerla posible y satisfactoria.

\section{REFERENCIAS CONSULTADAS}

Asamblea Nacional Constituyente del Ecuador. (2008). Constitución de la República del Ecuador. [Constitution of the Republic of Ecuador]. Obtenido de https://n9.cl/z0d0

Asamblea Nacional Constituyente del Ecuador. (2011). Ley Orgánica de Educación Intercultural. [Organic Law of Intercultural Education]. Obtenido de https://n9.cl/1fo3

Asamblea Nacional Contituyente del Ecuador. (2013). Código de la Niñez y la Adolescencia. [Code of childhood and adolescence]. Obtenido de https://n9.cl/fokqg

Banco Mundial. (2018). Educación. [Education]. Recuperado el 18 de septiembre de 2020, de Banco Mundial: https://n9.cl/4sok

Caira, B. (2014). Influencia de los padres de familia en el rendimiento académico de los alumnos de iii ciclo de educación primaria de la institución educativa jacob clerk maxwell del distrito de hunter. Arequipa: Universidad Nacional de San Agustín.

Calvo, N. (2011). Importancia de la Lectoescritura en casa. [Importance of Literacy at Home]. Recuperado el 16 de septiembre de 2020, de Blog de la Universidad de Burgos: https://n9.cl/r9c6m 
Universidad Nacional Experimental Francisco de Miranda (UNEFM). Santa Ana de Coro. Venezuela

María Gabriela Quezada-Padrón; Darwin Gabriel García-Herrera; Ana Zulema Castro-Salazar; Juan Carlos Erazo-Álvarez

Ceballos, E. y Rodrigo, M. (1998). La educación de los hijos. [The education of children]. Barcelona: Ediciones Crisol. Obtenido de https://n9.cl/vc06f

Darling, N., \& Steinberg, L. (2015). El estilo parental como contexto: un modelo integrador. [Parenting style as context: An integrative model]. Psychological bulletin, 83-95. Obtenido de https://n9.cl/xrgi

Edel, R. (2013). El rendimiento académico: concepto, investigación y desarrollo. [EI rendimiento académico: concepto, investigación y desarrollo]. REICE. Revista Iberoamericana sobre Calidad, Eficacia y Cambio en Educación, 1(2), 1-16.

Espinoza, E., \& Toscano, D. (2015). Metodología de Investigación Educativa y Técnica [Metodología de Investigación Educativa y Técnica]. Obtenido de https://n9.cl/7txl

Ferreiro, E., \& Teberosky, A. (1979). Los sistemas de escritura en el desarrollo del niño. [Writing Systems in child development]. México: Siglo XXI. Obtenido de https://n9.cl/o9j3p

Psacharopoulos, G., \& Patrinos, H. (2018). Rendimientos de la inversión en educación: una revisión decenal de la literatura mundial. [Returns to Investment in Education : A Decennial Review of the Global Literature]. Policy Research Working Paper. Recuperado el 17 de septiembre de 2020, de https://openknowledge.worldbank.org/handle/10986/29672

Rodrigo, M. y Palacios, J. (1998). Familia y Desarrollo Humano. [Family and Human Development]. Madrid: Alianza Editorial. Obtenido de https://n9.cl/fxto

Salinas, E. (2017). Influencia de los Padres de Familia en el Proceso de Aprendizaje de los niños de 0 a 3 años del Centro Creciendo con Nuestros Hijos, Comunidad Gañanzol. [Influence of the Parents in the Learning Process of the children from 0 to 3 years of the Center Grow]. Cuenca: Universidad Técnica Salesiana. Obtenido de https://n9.cl/hewm 